bttp://dx.doi.org/10.21707/gs.v11.n04a04

\title{
O AUMENTO DA TEMPERATURA REDUZ A TOLERÂNCIA AO ESTRESSE HÍDRICO NA GERMINAÇÃo DE SEMENTES DE PERESKIA GRANDIFOLIA Haw. subsp. GRANDIFOlda (CACtACEAE)?
}

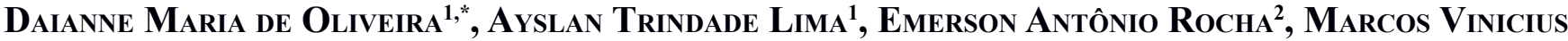 \\ Meiado ${ }^{1}$
}

${ }^{1}$ Laboratório de Fisiologia de Sementes, Departamento de Biociências, Universidade Federal de Sergipe. Av. Vereador Olimpio Grande, s/n, Bloco D, Campus Professor Alberto Carvalho, Bairro Porto, Itabaiana, Sergipe, Brasil. CEP: 49510-200.

${ }^{2}$ Departamento de Ciências Biológicas, Universidade Estadual de Santa Cruz, Rodovia Jorge Amado, Km 16, Salobrinho, Ilhéus, Babia, Brasil. CEP: $45662-900$.

*Autorpara correspondência: daiannemaria21@gmail.com

Recebido em 24 de outubro de 2016. Aceito em 14 de junho de 2017. Publicado em 30 de dezembro de 2017.

Resumo - O objetivo deste estudo foi avaliar se o aumento da temperatura reduz a tolerância ao estresse hídrico na germinação de sementes de Pereskia grandifolia Haw. subsp. grandifolia (Cactaceae). O déficit hídrico foi simulado com soluções de polietilenoglicol 6000 nos potenciais osmóticos de 0,$0 ;-0,1 ;-0,3 ;-0,6$ e - - ,9 MPa e as temperaturas constantes avaliadas foram as de 25 e $30^{\circ} \mathrm{C}$. Os dados de frequência da germinação de sementes indicaram diferenças no comportamento germinativo entre as temperaturas de 25 e $30^{\circ} \mathrm{C}$ em todos os potenciais avaliados. O potencial osmótico influenciou todos os parâmetros de germinação avaliados no presente estudo, com exceção do índice de sincronização. Com a redução do potencial osmótico foi constatada a redução da germinabilidade das sementes, independentemente da temperatura avaliada. Entretanto, foi observada uma redução mais drástica na germinabilidade quando as sementes foram submetidas ao déficit hídrico na temperatura de $30^{\circ} \mathrm{C}$. A temperatura também influenciou significativamente a germinabilidade das sementes, sendo observadas mais sementes germinadas na temperatura de $25^{\circ} \mathrm{C}$. O potencial básico foi de $-0,9005 \mathrm{MPa}$ na temperatura de $25^{\circ} \mathrm{C}$. Porém, quando as sementes foram submetidas à temperatura de $30^{\circ} \mathrm{C}$, o potencial básico aumentou para $-0,7540 \mathrm{MPa}$, indicando uma redução da tolerância ao déficit hídrico. Conclui-se que o aumento da temperatura reduz a tolerância ao estresse hídrico na germinação de sementes de $P$. grandifolia subsp. grandifolia e sugere-se um estudo com sementes de diferentes populações dessa espécie pra se confirmar a influência da origem da semente na sua resposta germinativa quando submetidas a estresses abióticos associados.

Palavras-chave: Cactos; Déficit Hídrico; Estresses Abióticos; Germinaç̃o; Sementes.

Does TEMPERATURE INCREASE REDUCE WATER STRESS TOLERANCE ON SEED GERMiNATION OF PERESKLA GR ANDIFOLIA Haw. SUBSP. GRANDIFOLIA (CACTACEAE)?

Abstract - The aim of this study was to evaluate if the temperature increase reduces the water stress tolerance on seed germination of Pereskia grandifolia Haw subsp. grandifolia (Cactaceae). The water stress was simulated with solutions of polyethylene glycol 6000 in osmotic potentials of $0.0,-0.1,-0.3,-0.6$ and $-0.9 \mathrm{MPa}$ and the constant temperatures evaluated were 25 and $30^{\circ} \mathrm{C}$. The data of relative frequency of seed germination indicated differences in germination behavior between 25 and $30^{\circ} \mathrm{C}$ in all evaluated potentials. The osmotic potential influenced all the germination parameters evaluated in the present study, with the exception of the synchronization index. With the reduction of the osmotic potential, the germinability was reduced, regardless of the temperature evaluated. However, a more drastic reduction in germinability 
was observed when the seeds were submitted to water stress at $30^{\circ} \mathrm{C}$. The temperature also influenced significantly the germinability of the studied species, being observed more seeds germinated in the temperature of $25^{\circ} \mathrm{C}$. The base water potential was $-0.9005 \mathrm{MPa}$ at the temperature of $25^{\circ} \mathrm{C}$. However, when the seeds were submitted to the temperature of $30^{\circ} \mathrm{C}$, the base water potential increased to $-0.7540 \mathrm{MPa}$, indicating a reduction in the water stress tolerance. We concluded that the increase in temperature reduces the water stress tolerance on seed germination of P. grandifolia subsp. grandifolia and we suggested a study with seeds of different populations of this species to confirm the influence of the seed origin on its germinative response when these seeds are submitted to associated abiotic stresses.

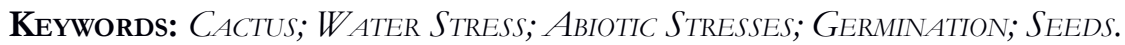

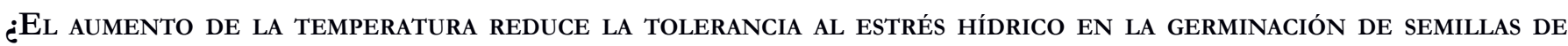
Pereskia grandifolia Haw. subsp. grandifolia (Cactaceae)?

Resumen: Se objetivó evaluar en este estudio si el aumento de la temperatura reduce la tolerancia al estrés hídrico en la germinación de semillas de Pereskia grandifolia Haw. subsp. grandifolia (Cactaceae). Se simuló el estrés hídrico con soluciones de polietilenglicol 6000 en los potenciales osmóticos de 0,$0 ;-0,1 ;-0,3 ;-0,6$ y - $0,9 \mathrm{MPa}$ y las temperaturas constantes evaluadas fueron las de 25 y $30^{\circ} \mathrm{C}$. Los datos de frecuencia de la germinación de semillas indicaron diferencias en el comportamiento germinativo entre las temperaturas de 25 y $30^{\circ} \mathrm{C}$ en todos los potenciales evaluados. El potencial osmótico influenció todos los parámetros de germinación evaluados en el presente estudio, con la excepción del índice de sincronización. Con la reducción del potencial osmótico se constató la reducción de la germinabilidad de las semillas, independientemente de la temperatura evaluada. Sin embargo, se observó una reducción más drástica en la germinabilidad cuando se sometieron las semillas al déficit hídrico a una temperatura de $30^{\circ} \mathrm{C}$. La temperatura también influenció significativamente la germinabilidad de las semillas, siendo observadas más semillas germinadas a la temperatura de $25^{\circ} \mathrm{C}$. El potencial osmótico base fue de $-0,9005 \mathrm{MPa}$ a una temperatura de $25^{\circ} \mathrm{C}$. Sin embargo, cuando se sometieron las semillas a una temperatura de $30^{\circ} \mathrm{C}$, el potencial osmótico base aumentó a $-0,7540 \mathrm{MPa}$, indicando una reducción de la tolerancia al estrés hídrico. Se concluye que el aumento de la temperatura reduce la tolerancia al estrés hídrico en la germinación de semillas de P. grandifolia subsp. grandifolia y se sugiere un estudio con semillas de diferentes poblaciones de esa especie para confirmar la influencia del origen de la semilla en su respuesta germinativa cuando son sometidas a estrés abióticos asociados.

Palabras clave: Cactus; Estrés Hídrico; Estrés Abiótico; Germinación; Semillas.

\section{INTRODUÇÃO}

Possuir a habilidade de germinar sob a influência de diferentes fatores abióticos é de extrema importância para as sementes, pois, assim, elas garantem sua sobrevivência e a regeneração das florestas (Ceccon et al. 2006, Moura et al. 2011). Essas unidades dispersivas apresentam grande diversidade de características adaptativas, que lhes permitem sobreviver também aos fatores físicos e às interações com outros seres vivos, como predação, alelopatia e competição (Perez et al. 2001, Moura et al. 2011). Dentre os fatores que influenciam diretamente a germinação das sementes está a diminuição do potencial hídrico do meio, que pode atrasar ou reduzir consideravelmente a germinabilidade de muitas espécies de plantas (Perez et al. 2001, Stefanello et al. 2006, Rego et al. 2011). Além disso, o período de germinação e o estabelecimento das plântulas são importantes para a sobrevivência das espécies, principalmente nos locais onde a disponibilidade de água é limitada durante certo período do ano (Rego et al. 2007).

Outro fator abiótico que também influencia direta e indiretamente a germinação das sementes em seu 
ambiente natural é a temperatura (Baskin e Baskin 2014). Essa influencia pode estar relacionada às alterações na velocidade das reações enzimáticas proporcionadas por temperaturas altas e baixas, bem como ao controle da cinética de absorção de água quando as sementes estão submetidas a essas temperaturas extremas (Castro et al. 2004, Baskin e Baskin 2014).

Apesar de o Brasil ser um dos três maiores centros de diversidade da família Cactaceae (Taylor e Zappi 2004, Zappi et al. 2011) poucos estudos são realizados com os cactos que ocorrem nos ecossistemas brasileiros (Meiado et al. 2015b). Os cactos constituem um grupo de recursos muito importantes para a fauna local (Rocha e Agra 2002, Rocha et al. 2007, Meiado et al. 2015b), porém, o manejo e a análise de sementes da maioria das espécies de plantas nativas do Nordeste ainda é insuficiente (Lima et al. 2006, Meiado et al. 2012).

Sabendo da importância das cactáceas, se faz necessário o estudo das espécies que constituem esse grupo, sendo o objetivo do presente trabalho, que avaliou o comportamento germinativo das sementes de Pereskia grandifolia Haw. subsp. grandifolia sob a influência de diferentes potenciais hídricos em duas temperaturas que as sementes podem ser submetidas em campo, contribuindo, assim, para o conhecimento ecofisiológico da espécie.

\section{Material e Métodos}

\section{Espécie estudada}

O gênero Pereskia Mill. (Cactaceae) apresenta distribuição neotropical e é representado no Brasil pela ocorrência de oito espécies (sete nativas e uma introduzida), das quais quatro são endêmicas e se distribuem por todas as regiões do país, com exceção da região Norte (Zappi et al. 2015). São encontradas espécies desse gênero em áreas de Caatinga, Cerrado, Mata Atlântica, Pampa e Pantanal e, diferente da maioria das espécies dessa família, os cactos do gênero Pereskia são arbustos, árvores ou lianas que apresentam folhas bem desenvolvidas em seus ramos, sendo decíduas na estação seca e, por vezes, suculentas (Taylor e Zappi 2004, Zappi et al. 2015).

Conhecida como ora-pro-nobis, a espécie Pereskia grandifolia Haw. subsp. grandifolia é uma planta que tem porte arbustivo/arbóreo e pode chegar a atingir de 2 a $10 \mathrm{~m}$ de altura. Seu tronco é espesso e esverdeado, com feixes de espinhos em sua extensão. As folhas são coriáceas simples e as flores estão dispostas em cimeiras terminais, com coloração rosa. Já seus frutos são do tipo baga piriforme e ficam verde-amarelados quando maduros (Corrêa 1984, Taylor e Zappi 2004). A espécie é nativa e endêmica do Brasil, com distribuição no Nordeste (Bahia, Ceará, Maranhão, Pernambuco, Piauí e Sergipe), Sudeste (Espirito Santo, Minas Gerais, Rio de Janeiro e São Paulo) e Sul (Paraná e Santa Catarina), compreendendo domínios de Caatinga e Mata Atlântica (Turra et al. 2008, Meiado et al. 2015a, Zappi et al. 2015).

\section{Interação entre temperatura e déficit hídrico}

Os frutos de P. grandifolia subsp. grandifolia foram coletados em áreas de Mata Atlântica do Estado da Bahia e levados para beneficiamento das sementes no Laboratório de Fisiologia de Sementes (LAFISE) da Universidade Federal de Sergipe, em Itabaiana, Sergipe. Para analisar o efeito da interação entre o déficit hídrico e a temperatura na germinação de sementes da espécie estudada foram definidos cinco potenciais hídricos e dois tratamentos de temperatura constante. O déficit hídrico foi simulado com soluções de polietilenoglicol 
(PEG 6000) nos seguintes potenciais: 0,0 (água destilada); -0,1; -0,3; -0,6 e -0,9 MPa (Villela et al. 1991) e as temperaturas constantes avaliadas foram as de 25 e $30^{\circ} \mathrm{C}$. Foram utilizadas quatro repetições de 25 sementes, que foram colocadas para germinar em placas de Petri de $9 \mathrm{~cm}$ de diâmetro, contendo duas folhas de papel filtro umedecidas com $4 \mathrm{~mL}$ das soluções de PEG 6000. As placas foram vedadas com filme de poliéster e mantidas nas temperaturas mencionadas anteriormente. $\mathrm{O}$ experimento foi avaliado diariamente, durante 20 dias e o critério utilizado para se considerar a germinação foi a protrusão da radícula.

\section{Parâmetros de germinação e análise estatística}

Ao termino do experimento foram calculados, no software GerminaQuant 1.0 (Marques et al. 2015), a germinabilidade $(\mathrm{G}=\%$ ), o tempo médio de germinação onde ti é o período desde o início do experimento até a enésima observação (dias) e ni é o número de sementes germinadas no tempo i (não o número acumulado, mas o número correspondente a enésima observação)], a velocidade média de germinação (VMG $=1 /$ t) e o índice de sincronização, onde fi é a frequência relativa da germinação (i.e., a proporção de sementes germinadas em intervalo)], bem como a frequência relativa de germinação $\left[f_{i}=n_{i} / \sum n_{i}\right]$ de acordo com Ranal e Santana (2006).

Para realizar a modelagem da germinação das sementes da espécie estudada foram calculados a taxa de germinação $\left(1 / \mathrm{t}_{50}\right)$, o potencial osmótico básico $\left(\mathrm{y}_{\mathrm{b}}-\mathrm{MPa}\right)$ e o tempo hídrico para germinação $\left(\mathrm{q}_{\mathrm{H}}-\mathrm{MPa}\right.$.dia). Para isso, em cada temperatura e potencial osmótico avaliado, a porcentagem final de germinação foi plotada como uma função de tempo e uma curva sigmoidal de Boltzmann foi ajustada no programa Origin ${ }^{\circledR}$ 9, a partir da qual o tempo para atingir de $10-90 \%$ de germinação da população foi estimado. A recíproca desses tempos (taxa de germinação) foi plotada contra o potencial osmótico. Regressões lineares em cada fração foram usadas para estimar a intercepção x e a inclinação de cada linha de regressão. Uma média das intercepções x resultou no potencial osmótico básico $\left(\mathrm{y}_{\mathrm{b}}\right)$, abaixo do qual as sementes não germinam (Gummerson 1986). Para cada tratamento também foi calculado o tempo hídrico para germinação através da fórmula $\mathrm{q}_{\mathrm{H}}=\left(\mathrm{y}-\mathrm{y}_{\mathrm{b}}\right) \cdot \mathrm{t}_{\mathrm{g}}$, na qual y corresponde ao potencial osmótico do meio, $\mathrm{y}_{\mathrm{b}}$ é o potencial osmótico básico e $\mathrm{t}_{\mathrm{g}}$ corresponde ao tempo desde o início da embebição (Gummerson 1986). Esse último parâmetro permite compreender e quantificar o quanto a germinação é modificada pela disponibilidade de água e descreve a relação entre o tempo de germinação e o potencial de água do meio (Cardoso 2013).

Os dados de germinabilidade foram transformados em arcoseno $\sqrt{\%} \%$ antes das análises estatísticas. A normalidade dos dados e a homogeneidade das variâncias foram verificadas através dos testes Shapiro-Wilk e Levene, respectivamente. Os resultados foram submetidos à ANOVA Dois Fatores (temperatura e potencial osmótico) e as médias foram comparadas pelo teste de Tukey a posteriori (Ranal e Santana 2006). Todas as análises estatísticas foram realizadas no programa STATISTICA 13 com índice de significância de 0,05 (StatSoft 2016).

\section{REsultados}

A germinação de P. grandifolia subsp. grandifolia teve início no terceiro dia após a semeadura, quando as sementes foram colocadas para germinar na solução de $0,0 \mathrm{MPa}$ e na temperatura de $25^{\circ} \mathrm{C}$ (Figura 1). Os dados de frequência da germinação de sementes da espécie estudada indicaram diferenças no comportamento germinativo entre as temperaturas de $25 \mathrm{e} 30^{\circ} \mathrm{C} \mathrm{em}$ todos os potenciais avaliados (Figura 1). Em $25^{\circ} \mathrm{C}$ e $0,0 \mathrm{MPa}$ 
foi observado um comportamento mais leptocúrtico da curva, com distribuição unimodal e maior porcentagem da frequência relativa de germinação em um menor espaço de tempo, indicando que esse foi o melhor tratamento para a germinação das sementes da espécie estudada (Figura 1).

Figura 1 - Frequência relativa diária (\%) da germinação de sementes de Pereskia grandifolia Haw. subsp. grandifolia (Cactaceae) submetidas a déficit hídrico nas temperaturas de 25 e $30^{\circ} \mathrm{C}$.
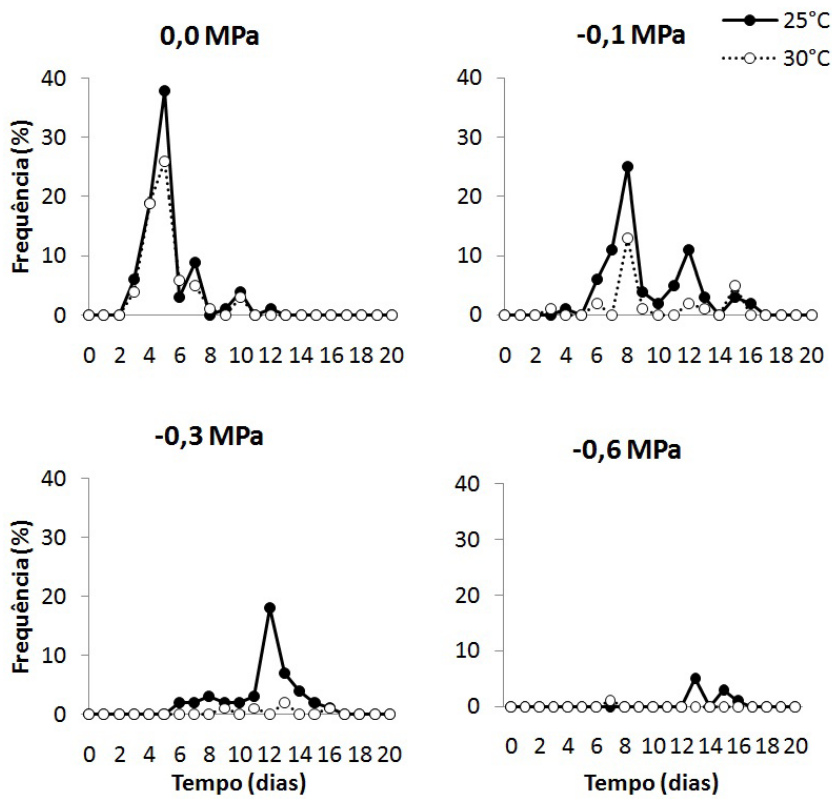

O potencial osmótico influenciou todos os parâmetros de germinação avaliados no presente estudo, com exceção do índice de sincronização (Tabela 1). Com a redução do potencial osmótico foi constatada a redução da germinabilidade das sementes de $P$. gradifolia subsp. grandifolia, independentemente da temperatura avaliada.

Tabela 1 - Tempo médio de germinação (TMG - dias), velocidade média de germinação (VMG - dias ${ }^{-1}$ ) e índice de sincronização (IS) da germinação de sementes de Pereskia grandifolia Haw. subsp. grandifolia (Cactaceae) submetidas a déficit hídrico nas temperaturas de 25 e $30^{\circ} \mathrm{C}$. Dados expressos em média \pm desvio padrão. Letras maiúsculas comparam o mesmo potencial osmótico em diferentes temperaturas e letras minúsculas comparam diferentes potenciais osmóticos em uma mesma temperatura.

\begin{tabular}{|c|c|c|c|c|c|}
\hline \multirow[b]{2}{*}{$25^{\circ} \mathrm{C}$} & \multicolumn{5}{|c|}{ Potencial Osmótico (MPa) } \\
\hline & 0,0 & $-0,1$ & $-0,3$ & $-0,6$ & $-0,9$ \\
\hline TMG & $5,3 \pm 0,5$ & $9,2 \pm 1,0$ & $11,5 \pm 0,7$ & $14,0 \pm 1,41$ & - \\
\hline (dias) & Ac & $\mathrm{Ab}$ & $\mathrm{Ab}$ & $\mathrm{Aa}$ & \\
\hline VMG & $0,19 \pm 0,02$ & $0,11 \pm 0,01$ & $0,08 \pm 0,01$ & $0,07 \pm 0,01$ & - \\
\hline$\left(\operatorname{dias}^{-1}\right)$ & Aa & $\mathrm{Ab}$ & Ac & Ac & \\
\hline \multirow[t]{2}{*}{ IS } & $0,28 \pm 0,06$ & $0,20 \pm 0,04$ & $0,20 \pm 0,05$ & $0,20 \pm 0,05$ & - \\
\hline & $\mathrm{Aa}$ & $\mathrm{Aa}$ & $\mathrm{Aa}$ & $\mathrm{Aa}$ & \\
\hline $30^{\circ} \mathrm{C}$ & 0,0 & $-0,1$ & $-0,3$ & $-0,6$ & $-0,9$ \\
\hline TMG & $5,1 \pm 0,2$ & $10,2 \pm 2,8$ & $12,5 \pm 1,9$ & $15,7 \pm 0,9$ & - \\
\hline (dias) & Ac & $\mathrm{Ab}$ & $\mathrm{Ab}$ & $\mathrm{Aa}$ & \\
\hline VMG & $0,19 \pm 0,01$ & $0,10 \pm 0,02$ & $0,08 \pm 0,01$ & $0,07 \pm 0,01$ & - \\
\hline$\left(\operatorname{dias}^{-1}\right)$ & $\mathrm{Aa}$ & $\mathrm{Ab}$ & $\mathrm{Ab}$ & $\mathrm{Ab}$ & \\
\hline \multirow[t]{2}{*}{ IS } & $0,26 \pm 0,02$ & $0,25 \pm 0,03$ & $0,25 \pm 0,02$ & $0,24 \pm 0,02$ & - \\
\hline & $\mathrm{Aa}$ & $\mathrm{Aa}$ & $\mathrm{Aa}$ & $\mathrm{Aa}$ & \\
\hline
\end{tabular}


Entretanto, foi observada uma redução mais drástica na germinabilidade quando as sementes foram submetidas ao déficit hídrico na temperatura de $30^{\circ} \mathrm{C}$ (Figura 2A), o que justifica a interação significativa entre os dois parâmetros avaliados (Tabela 1). O TMG e a VMG também foram influenciados pelo potencial osmótico (Tabelas 1 e 2).

Quando as sementes de P. gradifolia subsp. grandifolia foram colocadas para germinar no potencial de 0,0 $\mathrm{MPa}$, o TMG foi de 5,3 \pm 0,5 e 5,1 \pm 0,2 dias nas temperaturas de 25 e $30^{\circ} \mathrm{C}$, respectivamente (Tabela 2). Porém, em ambas as temperaturas, o TMG das sementes aumentou cerca de 100 e 300\% quando as sementes foram submetidas ao potencial osmótico de -0,1 e -0,6 MPa, respectivamente (Tabela 2). A VMG das sementes da espécie estudada também foi influenciada negativamente apenas pelo potencial osmótico. Independentemente da temperatura avaliada, foi observada uma redução na VMG com a redução do potencial osmótico (Tabelas 1 e 2).

Tabela 2 - Valor de F, grau de liberdade (gl) e índice de significância (p) dos parâmetros germinabilidade (G - \%), tempo médio de germinação (TMG - dias), velocidade média de germinação (VMG - dias ${ }^{-1}$ ) e índice de sincronização (IS) da germinação de sementes de Pereskia grandifolia Haw. subsp. grandifolia (Cactaceae) submetidas a diferentes potenciais hídricos e temperaturas e comparados pela ANOVA Dois Fatores.

\begin{tabular}{lccc}
\hline Fator & F & gl & P \\
\hline G (\%) & & & $<0,0001$ \\
Potencial & 126,456 & 4 & $<0,0001$ \\
Temperatura & 80,882 & 1 & $<0,0001$ \\
Potencial*Temperatura & 14,399 & 4 & $<0,0001$ \\
TMG (dias) & & & 0,0888 \\
Potencial & 66,719 & 3 & 0,6181 \\
Temperatura & 3,146 & 1 & \\
Potencial*Temperatura & 0,605 & 3 & $<0,0001$ \\
VMG (dias ${ }^{-1}$ ) & & & 0,4524 \\
Potencial & 123,435 & 3 & 0,9100 \\
Temperatura & 0,583 & 1 & \\
Potencial*Temperatura & 0,178 & 3 & 0,7873 \\
IS & & & 0,3568 \\
Potencial & 0,353 & 3 & 0,8660 \\
Temperatura & 0,883 & 1 & \\
Potencial*Temperatura & 0,242 & 3 & \\
\hline
\end{tabular}

A temperatura também influenciou significativamente a germinabilidade das sementes da espécie estudada, sendo observadas mais sementes germinadas na temperatura de $25^{\circ} \mathrm{C}$ (Figura $1 \mathrm{~A}$ e Tabela 1 ). Por outro lado, a temperatura não influenciou o TMG, a VMG, nem o IS da germinação das sementes de $P$. gradifolia subsp. grandifolia, não sendo observada interação entre os fatores potencial osmótico e temperatura nas análises desses parâmetros de germinação mencionados (Tabela 1).

De acordo com os modelos gerados com os dados de germinação das sementes da espécie estudada, o $\mathrm{y}_{\mathrm{b}}$ foi de $-0,9005 \mathrm{MPa}$ na temperatura de $25^{\circ} \mathrm{C}$. Porém, quando as sementes de P. gradifolia subsp. grandifolia foram submetidas à temperatura de $30^{\circ} \mathrm{C}, \mathrm{o}_{\mathrm{b}}$ aumentou pra $-0,7540 \mathrm{MPa}$, indicando uma redução da tolerância ao déficit hídrico (Figura 2B). Por sua vez, o $\mathrm{q}_{\mathrm{H}}$ observado na temperatura de $25^{\circ} \mathrm{C}$ foi de 4,9682 MPa.dia e de 4,4065 MPa.dia na temperatura de $30^{\circ} \mathrm{C}$. Na Figura $2 \mathrm{~B}$ foi possível observar um comportamento germinativo 
diferencial quando as sementes da espécie estudada foram submetidas ao déficit hídrico nas duas temperaturas avaliadas. Em $25^{\circ} \mathrm{C}$ foi observado uma maior taxa de germinação associada a uma maior tolerância ao déficit hídrico. Porém, quando as sementes da mesma espécie foram submetidas as déficit hídrico em $30^{\circ} \mathrm{C}$, foi possível observar uma redução da tolerância ao estresse e uma menor taxa de germinação, que está relacionada ao aumento do tempo de germinação (Figura 2B).

Figura 2 - (A) Germinabilidade (\%) e (B) taxa de germinação $\left(1 / \mathbf{t}_{50}\right)$ de sementes de Pereskia grandifolia Haw. subsp. grandifolia (Cactaceae) submetidas a déficit hídrico nas temperaturas de 25 e $30^{\circ} \mathrm{C}$. Dados estão representados na figura $2 \mathrm{~A}$ pela média \pm desvio padrão.

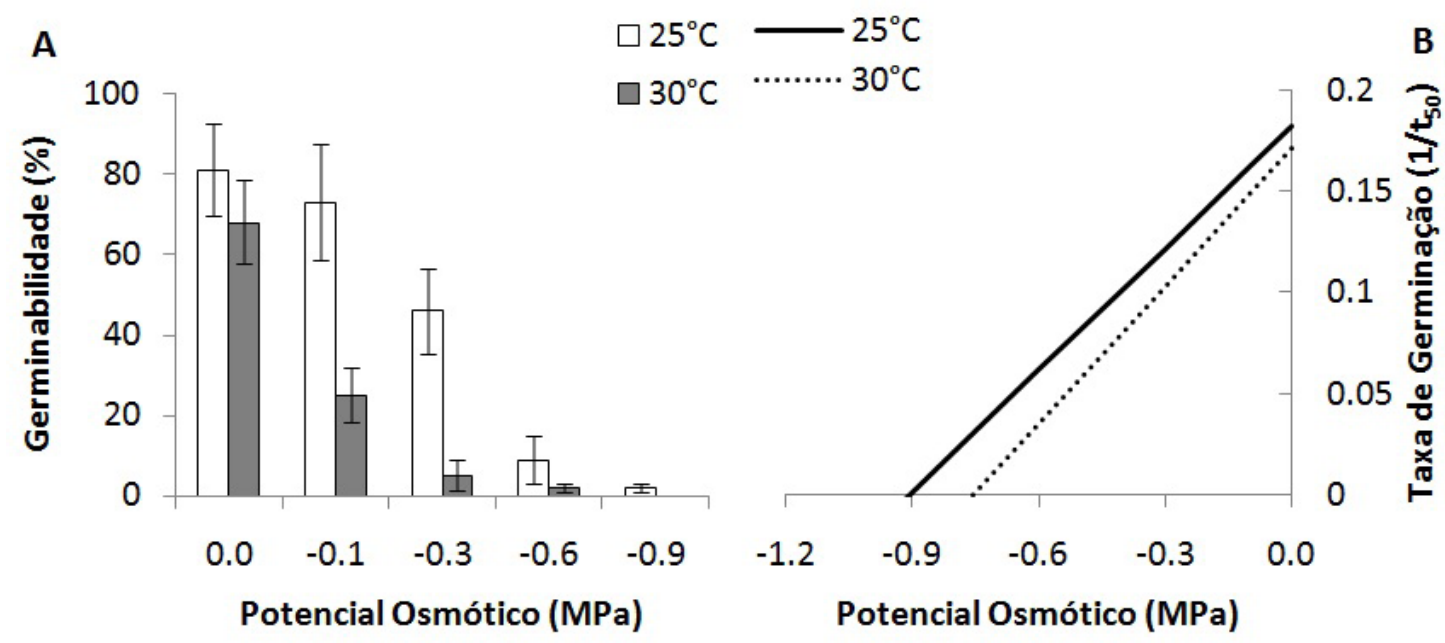

\section{Discussão}

Tolerar a seca é uma característica de grande importância quando se considera a recomendação de espécies vegetais capazes de suportar diferentes condições de potenciais osmóticos em diversas situações ecológicas, principalmente com baixa disponibilidade hídrica, como ocorre com espécies encontradas na Caatinga, a exemplo a P. grandifolia subsp. grandifolia. Rosa et al. (2005) ressaltaram que algumas espécies florestais cujas sementes chegam a germinar mesmo em condições de déficit hídrico apresentam vantagens ecológicas quando comparadas com outras que são vulneráveis à seca. Assim, a distribuição geográfica das espécies se dá pela capacidade que suas sementes possuem de germinar em diversas condições climáticas, se estabelecendo no clima que melhor se adaptam (Borghetti e Ferreira 2004).

Cada espécie germina em um valor de potencial hídrico critico especifico e, abaixo deste, a germinação não ocorre (Carvalho 2005). Como visto nos resultados deste estudo, o limite de tolerância para germinação da P. grandifolia subsp. grandifolia quando utilizado o agente osmótico PEG, foi reduzido com o aumento da temperatura. Tais resultados condizem com os encontrados na literatura, pois a concentração do PEG em meio de germinação inibiu a absorção de água pelos tecidos, impedindo que a atividade metabólica seja retomada pela semente e dificultando, assim, o início da germinação. Em condições naturais, o estresse hídrico pode atuar de forma positiva no estabelecimento das espécies, provocando um atraso significativo no tempo de germinação das sementes (Fanti e Perez 2004). Assim, a germinação é distribuída no tempo e espaço e as espécies podem se estabelecer ao encontrar condições favoráveis para esse processo (Bewley e Black 1994).

Com a diminuição do potencial osmótico, a absorção de água se torna mais lenta (Stefanello et al. 2006). A semente acaba iniciando a germinação e, não havendo água suficiente para dar continuidade, o processo é 
cessado, podendo haver até a morte do embrião, reduzindo a porcentagem final e a velocidade de germinação, além de aumentar o tempo médio de germinação e alterar a sincronia (Stefanello et al. 2006). Resultados semelhantes foram encontrados neste estudo, onde praticamente todos os parâmetros de germinação avaliados foram influenciados negativamente pela redução do potencial osmótico. Essa diminuição na germinação das sementes submetidas ao déficit hídrico é determinada pela redução das atividades enzimáticas, que promovem menor desenvolvimento do meristema (Santos et al. 1992).

Como visto nos resultados do presente estudo, as alterações no processo germinativo das sementes também são influenciadas pelo aumento da temperatura. Embora Souza et al. (2016) tenham indicado a temperatura constante de $33^{\circ} \mathrm{C}$ como sendo a temperatura ideal para a germinação das sementes da espécie P. grandifolia subsp. grandifolia, foi possível observar nos resultados do presente estudo que o aumento da temperatura de 25 para $30^{\circ} \mathrm{C}$ proporcionou uma redução da tolerância ao déficit hídrico, confirmado pelo aumento do $\mathrm{y}_{\mathrm{b}}$. e a consequente alteração do $\mathrm{q}_{\mathrm{H}}$. As diferenças observadas em relação à resposta das sementes dessa espécie sob a influência da temperatura podem estar relacionada a dois fatores distintos. O primeiro deles seria a diferença observada entre sementes produzidas em diferentes populações, as quais são influenciadas diretamente pelas características edafoclimáticas do ambiente que se encontram as plantas parentais no período de reprodução. Já o segundo seria o fato de que, no presente estudo, se analisou a interação entre dois tipos de estresses aos quais as sementes são submetidas constantemente na natureza, os estresses hídrico e térmico.

De maneira oposta aos resultados que foram observados neste estudo, algumas espécies arbóreas produzem sementes cuja tolerância ao estresse hídrico aumenta com a alteração da temperatura de 25 para $30^{\circ} \mathrm{C}$. Com o aumento da temperatura, sementes de pau-de-jangada [Apeiba tibourbou Aubl. (Malvaceae)] apresentaram um aumento de 14 e 9\% na germinabilidade quando submetidas aos potenciais osmóticos de -0,2 e -0,4 $\mathrm{MPa}$, respectivamente (Guedes et al. 2013). Outros parâmetros de germinação e de desenvolvimento inicial como, por exemplo, o índice de velocidade de germinação, o comprimento e a biomassa seca das plântulas também são favorecidos pelo aumento da temperatura quando as sementes são submetidas a estresse hídrico. Segundo Guedes et al. (2013), plântulas de pau-de-jangada em condições de déficit de -0,4 MPa produziram cerca do dobro de biomassa em $30^{\circ} \mathrm{C}$ quando comparadas com plântulas que se desenvolveram em $25^{\circ} \mathrm{C}$ no mesmo potencial osmótico. Assim, estresses associados podem alterar o comportamento germinativo das sementes e plântulas e se aproximar ainda mais da realidade das condições ambientais que estas enfrentam diariamente para conseguir completar seu processo germinativo e se estabelecer na natureza, influenciando, diretamente, a distribuição espacial das espécies de plantas no ambiente (Baskin e Baskin 2014).

\section{Conclusão}

Conclui-se que o aumento da temperatura reduz a tolerância ao estresse hídrico na germinação de sementes de $P$. grandifolia subsp. grandifolia e sugere-se um estudo com sementes de diferentes populações dessa espécie para se confirmar a influência da origem da semente na sua resposta germinativa quando submetidas a estresses abióticos associados.

\section{REFERÊNCIAS}


Baskin CC e Baskin JM. 2014. Seeds: Ecology, Biogeography, and Evolution of Dormancy and Germination. San Diego: Academic Press, 1586 p.

Bewley JD e Black M. 1994. Seeds: Physiology of Development and Germination. New York: Plenum Press, 445 p.

Borghetti F e Ferreira AG. 2004. Interpretação de resultados de germinação. In: Ferreira AG e Borghetti F (Eds.), Germinação: do básico ao aplicado. Porto Alegre: Artmed, p. 209-222.

Cardoso VJM. 2013. Os modelos hydrotime e hydrothermal time na análise da germinação em função da temperatura e potencial de água. Oecologia Australis, 17(3): 347-357.

Carvalho CJR. 2005. Respostas de plantas de Schizolobium amazonicum [S. parabyba var. amazonicum] e Schizolobium parabyba [Schizolobium parabybum] à deficiência hídrica. Revista Árvore, 29(6): 907-914.

Castro RD, Bradford KJ e Hilhorst HWM. 2004. Embebição e reativação do metabolismo. In: Ferreira AG e Borghetti F (Orgs.), Germinação: do básico ao aplicado. Porto Alegre: Artmed, p. 149-162.

Ceccon E, Huante P e Rincon E. 2006. Abiotic Factors Influencing Tropical Dry Forests Regeneration. Brazilian Archives of Biology and Technology, 49(2): 305-312.

Corrêa MP. 1984. Dicionário de plantas uteis do Brasil e exóticas cultivadas. Rio de Janeiro: Ministério da Agricultura do Rio de Janeiro.

Fanti SC e Perez SCJGA. 2004. Processo germinativo de sementes de paineira sob estresses hídrico e salino. Pesquisa Agropecuária Brasileira, 39(9): 903-909.

Guedes RS, Alves EU, Viana JS, Gonçalves EP, Lima CR e Santos SRN. 2013. Germinação e vigor de sementes de Apeiba tibourbou submetidas ao estresse hídrico e diferentes temperaturas. Ciência Florestal, 23(1): 45-53.

Gummerson RJ. 1986. The Effect of Constant Temperature and Osmotic Potentials on the Germination of Sugar Beet. Journal of Experimental Botany, 37(179): 729-741.

Lima JD, Almeida CC, Dantas VAV, Silva BMDS e Moraes WDS. 2006. Effect of Temperature and Substrate on Seed Germination of Caesalpinia ferrea Mart. ex Tul. (Leguminosae, Caesalpinoideae). Revista Árvore, 30(4): 513-518.

Marques FRF, Meiado MV, Castro NMCR, Campos MLO, Mendes KR, Santos OO e Pompelli MF. 2015. GerminaQuant: A New Tool for Germination Measurements. Journal of Seed Science, 37(3): 248-255.

Meiado MV, Aona LYS, Nascimento JPB, Lima AT e Zappi DC. 2015a. Cactaceae. In: Prata APN, Farias MCV e Landim MF (Orgs.), Flora de Sergipe. Vol. 2. Aracaju: Gráfica Editora Triunfo, p. 115-142.

Meiado MV, Machado MC, Zappi DC, Taylor NP e Siqueira Filho JA. 2015b. Ecological Attributes, Geographic Distribution and Endemism of Cacti from the São Francisco Watershed. Gaia Scientia, 9(2): 40-53.

Meiado MV, Silva FFS, Barbosa DCA e Siqueira Filho JA. 2012. Diaspores of the Caatinga: A Review. In: Siqueira 
Filho JA (Org.), Flora of the Caatingas of the São Francisco River - Natural History and Conservation. Rio de Janeiro: Andrea Jakobsson Estúdio Editorial, p. 306-365.

Moura MR, Lima RP, Farias SGG, Alves AR e Bezerra R. 2011. Efeito do estresse hídrico e do cloreto de sódio na germinação de Mimosa caesalpiniifolia Benth. Revista Verde de Agroecologia e Desenvolvimento Sustentável, 6(2): 230-235.

Perez SCJGA, Fanti SC e Casali CA. 2001. Influência da luz na germinação de sementes de canafístula submetidas ao estresse hídrico. Bragantia, 60(3): 155-156.

Ranal MA e Santana DG. 2006. How and Why to Measure the Germination Process? Revista Brasileira de Botânica, 29(1): 1-11.

Rego SS, Ferreira MM, Nogueira AC e Grossi F. 2007. Influência de potenciais osmóticos na germinação de sementes de Anadenanthera colubrina (Veloso) Brenan (Angico-branco) - Mimosaceae. Revista Brasileira de Biociências, 5(2): 549-551.

Rego SS, Ferreira MM, Nogueira AC, Grossi F, Sousa RK, Brondani GE e Silva ALL. 2011. Estresse hídrico e salino na germinação de sementes de Anadenanthera colubrina (Veloso) Brenan. Journal of Biotechnology and Biodiversity, 2(4): 37-42.

Rocha EA e AGRA MF. 2002. Flora do Pico do Jabre, Paraiba, Brasil: Cactaceae Juss. Acta Botanica Brasilica, 16(1): 15-21.

Rocha EA, Machado IC e Zappi DC. 2007. Floral Biology of Pilosocereus tuberculatus (Werderm.) Byles \& Rowley: A Bat Pollinated Cactus Endemic from the "Caatinga" in Northeastern Brazil. Bradleya, 25(1): 129-144.

Rosa LSD, Felippi M, Nogueira AC e Grossi F. 2005. Avaliação da germinação sob diferentes potenciais osmóticos e caracterização morfológica da semente e plântula de Ateleia glaz̧ioviana Baill (timbó). Revista Cerne, 11(3): 306-314.

Santos VD, Calil AC, Ruiz HA, Alvarenga EM e Santos CD. 1992. Efeito do estresse salino e hídrico na germinação e vigor de sementes de soja. Revista Brasileira de Sementes, 14(2): 189-194.

Souza LF, Gasparetto BF, Lopes RR e Barros IBI. 2016. Temperature Requirements for Seed Germination of Pereskia aculeata and Pereskia grandifolia. Journal of Thermal Biology, 57(1): 6-10.

StatSoft. 2016. STATISTICA 13. StatSoft South America. Available from URL: http://www.statsoft.com.br. Cited 21 May 2016.

Stefanello R, Garcia DC, Menezes ND, Muniz MFB e Wrasse CF. 2006. Efeito da luz, temperatura e estresse hídrico no potencial fisiológico de sementes de funcho. Revista Brasileira de Sementes, 28(2): 135-141.

Taylor N e Zappi D. 2004. Cacti of Eastern Brazil. Kew: Royal Botanic Gardens, 499 p.

Turra AF, Marçal FJB, Baretta IP, Takemura OS e Laverde-Jr A. 2008. Avaliação das propriedades antioxidantes 
e susceptibilidade antimicrobiana de Pereskia grandifolia Haworth (Cactaceae). Arquivos de Ciências da Saúde da UNIPAR, 11(1): 9-14.

Villela FA, Doni Filho L, Sequeira LL. 1991. Tabela de potencial osmótico em função da concentração de polietileno glicol 6.000 e da temperatura. Pesquisa Agropecuária Brasileira, 26(12): 1957-1968.

Zappi D, Taylor N, Santos MR e Larocca J. 2015. Cactaceae. In: Lista de Espécies da Flora do Brasil. Rio de Janeiro: Jardim Botânico do Rio de Janeiro. Disponível em: http://floradobrasil.jbrj.gov.br/jabot/floradobrasil/ FB1636.

Zappi D, Taylor N, Silva SR e Machado M. 2011. Plano de ação nacional para conservação das Cactáceas. Brasília: Instituto Chico Mendes de Conservação da Biodiversidade. 